preprint LBL-20131

\title{
Geomagnetic Reversals
}

Driven by

\section{Abrupt Sea Level Changes}

\author{
by \\ Richard A. Muller \\ and \\ Donald E. Morris \\ Lawrence Berkeley Laboratory \\ University of California \\ Berkeley, California 94720
}

27 August 1985

\begin{abstract}
Changes in the moment of inertia of the earth, brought about by the redistribution of ocean water from the tropics to ice at high latitudes, couple energy from the spin of the earth into convection in the liquid core. This mechanism may help provide the driving energy for the earth's dynamo. Sufficiently rapid ocean level changes can disrupt the dynamo, resulting (in half of the cases) in a geomagnetic field reversal. The model can account for the previously mysterious correlation reported between geomagnetic reversals and mass extinctions.
\end{abstract}


The most successful theory to account for the magnetic field of the earth is the dynamo model suggested by J. Larmor ${ }^{1}$ and first analyzed in detail by W. M. Elsasser ${ }^{2}$ and E. C. Bullard. ${ }^{3}$ Progress in this theory has recently been reviewed by R. T. Merrill and M. W. McElhinny. ${ }^{4}$ Although the model qualitiatively accounts for many of the main features of the Earth's magnetic field, there are still substantial problems. The most important of these is the fact that no one has yet found a self-consistent mathematical solution that actually gives the observed field. Although the source of energy for the dynamo is believed to be convection in the liquid core of the earth, it is not known what the source of energy for this convection is. 5 Proposed sources include radioactive decay, primordial energy from the collapse of the earth, and the latent heat of fusion from the still solidifying core. It is also difficult to account for the frequent geomagnetic reversals, which have occurred at a rate as high as 5/Myr, although T. Rikitake 6 and others have been able to reproduce this behavior qualitatively by assuming the liquid core incorporates two or more weakly coupled dynamos. A summary of work on reversals has recently been published by J. A. Jacobs. 7

Our present work was stimulated by the analysis by D. Raup ${ }^{8}$ of a correlation between the rate of geomagnetic reversals and the mass extinctions, which built upon the work of J. Negi and R. Tiwari ${ }^{9}$ and others. ${ }^{7}$ In our view there has been convincing evidence presented by L. Alvarez and coworkers 10 that several mass extinctions have been triggered by the impacts of comets or asteroids. A bolide $3 \mathrm{~km}$ in diameter moving at 25 $\mathrm{km} / \mathrm{sec}$ with respect to the earth impacts with an energy of $10^{29} \mathrm{ergs}$, comparable to the energy stored in the earth's magnetic field. But we were unable to find a mechanism that could directly couple the energy of the impact into that of the dynamo flow field. However we did find a mechanism whereby the impact can cause energy first to be transferred to the rotation of the mantle, and then to the fluid core, disrupting the dynamo action. The model has implications that are important even if the correlations seen by Raup are not substantiated. In the remainder of this paper we will explain this mechanism.

Consider a sudden sea level drop of $\mathrm{d}_{\mathrm{W}}=10$ meters taking place in a few hundred years. Sea level changes of this magnitude or greater have occurred at least 41 times in the last 65 million years, $12,13,14$ although the actual rate of change is unresolved and thus uncertain. For simplicity, assume that all of the water is deposited as snow and ice in the temperate and polar zones (|latitude $\mid>45^{\circ}$ ) where its contribution to the Earth's moment of 
inertia is substantially smaller. (The present south polar ice cap probably contains sufficient ice to raise the ocean levels by 50 to 100 meters, should it melt.) Prior to its removal (and neglecting details of geography) the shell of water had a moment of inertia $\mathrm{I}_{\mathrm{W}}$ of

$$
\mathrm{I}_{\mathrm{W}}=(2 / 3) \mathrm{Mr}_{\mathrm{e}}^{2}=(8 \pi / 3) \rho_{\mathrm{w}} \mathrm{r}^{4} \mathrm{~d}_{\mathrm{w}}
$$

where $M$ is the mass of the water, $\rho_{\mathrm{w}}$ is its density $\left(=1 \mathrm{gm} / \mathrm{cm}^{2}\right)$, and $\mathrm{r}_{\mathrm{e}}$ is the radius of the earth $\left(=6.4 \times 10^{8} \mathrm{~cm}\right)$. From the conservation of angular momentum, the sudden displacement of this water closer to the earth's axis will cause an increase in the angular velocity $\omega$ of the crust and mantle by an amount

$$
\Delta \omega / \omega=\left(\mathrm{I}_{\mathrm{w}} / \mathrm{I}_{\mathrm{m}}\right)
$$

The angular momentum of the mantle is $I_{m}=(8 \pi / 3) \rho_{m} r_{m}{ }^{4} d_{m}$, where $\rho_{m}$ is the density of the mantle (about $5 \mathrm{~g} / \mathrm{cm}^{3}$ ) and $r_{m}$ is the average radius of the mass of the mantle (weighted appropriately with $\mathrm{r}^{4}$ ), approximately $5.8 \times 10^{8} \mathrm{~cm}$, and $\mathrm{d}_{\mathrm{m}}$ is its thickness $\left(3 \times 10^{8} \mathrm{~cm}\right)$. Putting all these equations together and substituting values, we find

$$
\begin{aligned}
\Delta \omega & =\omega\left(\mathrm{I}_{\mathrm{w}} / \mathrm{I}_{\mathrm{m}}\right)=\omega\left(\rho_{\mathrm{w}} / \rho_{\mathrm{m}}\right)\left(\mathrm{r}_{\mathrm{e}} / \mathrm{r}_{\mathrm{m}}\right)^{4}\left(\mathrm{~d}_{\mathrm{w}} / \mathrm{d}_{\mathrm{m}}\right) \\
& =10^{-13} \mathrm{~d}_{\mathrm{w}}
\end{aligned}
$$

For $\mathrm{d}_{\mathrm{w}}=10$ meters $=10^{3} \mathrm{~cm}$, we find $\Delta \omega=10^{-10} / \mathrm{sec}$. This is a factor of 30 larger than the typical fluctuations seen in the rotation rate of the earth over periods of several decades. The entire mantle will pick up this motion relatively quickly. At the bottom of the mantle the velocity from the increased spin is $r_{c} \Delta \omega=0.03 \mathrm{~cm} / \mathrm{sec}$, where $r_{c}$ is the core radius.

The solid core of the earth is decoupled from the mantle by the liquid core. Thus the liquid will experience a shear, resulting in turbulent motion (because of the high Reynold's number). The existing magnetic field serves to couple the motion of the inner parts of the core to the outer parts, a phenomena sometimes referred to as "magnetic viscosity." Eddies will gradually transfers angular momentum from the mantle to the outer liquid core, then to the inner liquid core and finnaly to the solid core. The velocities induced in the liquid core will have a magnitude comparable to the shear velocity, i.e. roughly 0.03 $\mathrm{cm} / \mathrm{sec}$, until the solid core is spun up to match the spin of the mantle and the turbulent 
eddies damp out.

We would like to compare this new velocity $(0.03 \mathrm{~cm} / \mathrm{sec})$ to the velocities $\mathrm{v}$ of liquid flow previously existing in the dynamo. Unfortunately the flow velocities in the dynamo are not directly measureable, and their predicted values are somewhat model dependent. However we can estimate their rough magnitude from the following considerations. In order for the dynamo process to take place, the velocities must be sufficiently high that the magnetic field is carried along by the moving fluid, yet sufficiently low that significant diffusion takes place. This requirement implies that the "magnetic Reynold's number" $R_{m}=(v \tau / L) \geq 10$, where $\tau$ is the free decay time of the magnetic field (about $10^{4} \mathrm{yr}$ ) and $\mathrm{L}$ is the thickness of the liquid core. This equation implies that $\mathrm{v} \geq 10^{-2} \mathrm{~cm} / \mathrm{sec}$. This is approximately the same value that one obtains by assuming that the westward drift of the non-dipole component of the geomagnetic field, about 0.17 degrees per year, is due to transport of field by conductor moving with the velocity $\mathrm{v}$.

Thus the flow induced by the shear between the mantle and the solid core sets up a flow field in the liquid core whose magnitude is comparable to that of the previously existing flow field. This new motion through the existing magnetic field will result in electric currents which do not necessarily conform to the geometry necessary for dynamo action.

The magnetic field will attempt to prevent the outer liquid core from being accelerated with respect to the inner solid core, but the energy being transfered to the outer core by the mantle, $U_{\Delta \omega}=I_{c} \omega \Delta \omega=10^{29}$ erg, is comparable to the energy $U_{H}$ stored in the magnetic field of the dynamo (assuming 100 gauss over the volume of the core; $\mathrm{I}_{\mathrm{c}}$ is the moment of inertia of the outer core). Therefore the stored energy in the magnetic field is insufficient to prevent the change in the velocity field from taking place. The "feedback loop" necessary to maintain the dynamo will be disrupted, and the dynamo will turn off in a period comparable to the free-decay time 4,000 to 10,000 years for the dipole component. In a comparable period a new dynamo will build up, at first conforming to the new velocity fields, but then gradually transforming them as the energy in the field becomes signficant itself. The new magnetic field has only a 50\% probability of aligning 
in the same orientation as the previous field (assuming that the dynamo was completely disrupted), so in half the cases we expect to see a magnetic field reversal. In the other half we should see an "excursion" or "aborted reversal," i.e. a reversal that seemed to start, only to collapse, all within a period of a few times the magnetic diffusion time through the liquid core.

It is interesting to note how spin-up of the mantle actually takes place. As water from the relatively warm oceans continues to evaporate near the equators and begins to travel north (and south), the coriolis force causes the migration to turn into cyclones. These cyclones rotate in the proper directions (clockwise in the south, counter-clockwise in the north) to speed up the rotation of the earth as they precipitate out. Similar cyclonic flows in the ocean water could likewise transfer angular momentum to the earth as the water from the equator flows towards the poles to replace evaporated water from higher lattitudes. (Water near the equator may be replenished at a higher rate than in the north if the rivers in this region do not freeze.) Thus weather (and the sun) are the ultimate source of energy to turn off the dynamo.

Note that a sudden rise in the sea level can have a similarly disruptive effect on the geomagnetic field. If the Antarctic ice shelf is truly unstable, as some speculate, then global warming (perhaps from $\mathrm{CO}_{2}$ increases in the atmosphere) may trigger its slide from the continent into the sea. If that happens we will have the opportunity to test our theory directly by watching the slow-down of the earth's spin and the beginning of the turnoff of the geomagnetic field. We do not recommend that this experiment be carried out to test our prediction.

The disrupting velocity field applied by the mantle to the outer parts of the core, $\mathrm{v}=$ $\omega \mathrm{r}_{\mathrm{e}} \cos (\theta)$, is proportional to the first order spherical harmonic $\mathrm{Y}_{\mathrm{O}}{ }^{1}$. It is plausible that this simple velocity field could disrupt the lowest order component of the dynamo, the dipole field, while leaving the higher order components unaffected, in agreement with the observed multipole structure seen during magnetic field reversals. Detailed investigation of this point requires a detailed dynamo model.

The fact that the velocities induced in the core are comparable to the dynamo velocities suggests that our mechanism may be an important contributor to the energy that drives the dynamo. We know that sea level recessions of roughly 10 meters occur every 
two million years or more, and that some regressions are much larger, exceeding 100 meters. The induced velocities will endure until the solid core reaches the new transverse velocity of the mantle, or until the mantle slows down (through melting of the ice caps) to its previous velocity. The mean-square velocity contribution from this mechanism appears sufficient to keep the dynamo in operation by itself for substantial periods of time. It is important in this model that the coherent velocity changes experienced by the mantle and solid core are randomized through turbulent action in the liquid core, and persist until the core velocity matches that required to keep the system in equilbrium. (Equilibrium is not reached when the outer edge of the solid core and the inner edge of the mantle have the same angular velocity $\omega$, but when they have the same transverse velocity $v_{\phi}=\omega \mathrm{r}$. Material convecting between the two surfaces changes $\omega$ as $1 / \mathrm{r}$ to conserve angular momentum.)

The correlation reported by Raup ${ }^{8}$ between the magnetic reversals and the mass extinctions can now be explained with the following model. When a sufficiently large comet or asteroid strikes the earth, the material lofted from the crater darkens the high altitude sky, in the manner first discussed by Alvarez et al. ${ }^{10}$ Calculations by Toon et al. ${ }^{11}$ show that the dust, by serving as an intermediate absorber and radiator, will lower the temperatures of the continental land masses below freezing. Due to their high heat caAfter the dust settles, it is plausible to assume that the reduced albido from the large areas of land covered with ice and snow will cause the ice age to persist, although we have no detailed calculations to substantiate this assumption. If, in fact, the climate change persists then the oceans would transfer the 10-meter-thick layer of water to ice at northern lattitudes in a period of a few hundred years. The fact that this period is shorter than the time for momentum transfer across the Earth's liquid core layer is necessary in order for the 0.03 $\mathrm{cm} / \mathrm{sec}$ velocities to be transfered from the mantle to the fluid.

Note that we assume that mass extinctions triggered by impacts have a high probability of being accompanied by sea level changes. Previously noted coincidences between mass extinctions and sea level regressions have led some paleontologists to speculate that the sea level regression caused the extinctions, although we see here that it is possible that the extinctions and the regressions were simply caused by the same agent, the bolide impact. If we accept the hypothesis that some of the impacts occur in comet showers lasting a few million years $15,16,17$ then we see that regressions caused by impacts early in the showers could appear to anticipate extinctions caused by impacts later 
in the shower. Note that we make no claim that impacts caused all sea level regressions, or that all impacts will be accompanied by such regressions. Other effects could certainly lower the sea surface, including changes in the intensity of the sun, large volcanic eruptions, and auto-catalytic changes in the albido of the Earth.

One possible difficulty with the model is the fact that the number of observed reversals is much larger (over 120 in the last $65 \mathrm{Myr}$ ) than the number of identified abrupt sea level regressions (41) for the same period. There are several possible ways out of this difficulty. First, it is possible that not all the relevant sea level regressions have been found, since a regression can be missed if it takes place during a period of high geologic activity. Second, another mechanism may be contributing to the reversals, such as weakly-coupled multiple-dynamos in the core. And finally, the mechanism depends on the transfer of water to non-floating ice near the poles. Unless there is land mass near the poles or the ice is deep enough to create an ice continent, we would have floating ice, which does not change the moment of inertia of the oceans sufficiently to affect the dynamo. We offer no new solution to the previously noted paradox 8 that no evidence for glaciation has been found at the times of many of the sudden sea level regressions.

The theory we present here makes several major predictions. If the sudden change in the moment of the earth is the sole cause of geomagnetic reversals, then there should be an equal number of reversals and aborted reversals. In contrast, the coupled dynamo model predicts very few aborted reversals. If both mechanisms contribute to the observed reversals then all we can say is that there should be at least as many aborted reversals as half the number of sudden sea level regressions. The most important prediction of the theory is the strong correlation that should exist between the sea level regressions and the geomagnetic reversals. Unfortunately the time scales for the two sets of data must be calibrated with respect to each other to a resolution finer than a fraction (say 30\%) of the mean time between reversals, i.e. to 100,000 years or better, before the correlation can be attempted. At present this calibration does not exist, in fact many of the dates of the published sea level regressions have been rounded to the nearest half million years.

There have been many reports of correlations between the Earth's magnetic field and climate, and an entire chapter of Jacobs' book ${ }^{7}$ it devoted to them. The correlations seen are, at best, disputed. However our prediction is different in a fundamental way from the effects others have searched for. We do not predict short-term correlations between climate and magnetic field variations. Rather we predict that abrupt changes in sea level 
will be shortly followed by an excursions or reversal of the field. If the sea level change is suffiently slow (as has certainly been the case for historical periods) then we know of no observable effect. Unfortunately we do not know enough about the Earth's dynamo to be able to make a precise prediction of the minimum rate of sea level change to trigger a reversal or excursion. For example, if the normal fluid velocities in the dynamo are substantially greater than the $10^{-2} \mathrm{~cm} / \mathrm{sec}$ that we assumed, then a faster drop or rise in sea level ( $>10$ meters in a few hundred years) is required to affect it.

Walter Alvarez pointed out to us that a well-documented sudden sea level change took place just over 5 million years ago, when the dried-out Mediterranean was flooded with ocean water. ${ }^{18}$ The sudden redistribution of sea water at this time may have been large and sudden enough to have affected the Earth's moment of inertia. It would be worthwhile attempting to obtain an accurate date for this flooding to see if a reversal or excursion actually occurred at that time.

If no further correlation is found to exist between sudden sea-level changes and geomagnetic reversals, then we have a disturbing puzzle. It is difficult to see how a sufficiently large and sudden sea-level change could avoid severely affecting the Earth's field, unless the dynamo theory of the geomagnetic field is incorrect.

\section{Acknowledgments}

The authors are grateful to W. Alvarez, W. Press, R. Jeanlow, and P. Vail for helpful conversations.

\section{References}

1. J. Larmor, Rep. Brit. Ass. pp. 159-160 (1919).

2. W. M. Elsasser, Phys. Rev. 69, 106-116 (1946)

3. E. C. Bullard, Proc. R. Soc. London Ser. A, 197, 433-453 (1949).

4. R. T. Merrill and M. W. McElhinny, The Earth's Magnetic Field (Academic Press, 1983).

5. D. Gubbins and T. G. Masters, Adv. Geophys. 21, 1 (1979).

6. T. Rikitake, Proc. Cambridge Philos. Soc. 54, 89-105 (1958).

7. J. A. Jacobs, Reversals of the Earth's magnetic field (Adam Hilger Ltd, 1984).

8. D. M. Raup, Nature 314, 341-342 (1985). 
9. J. G. Negi and R. K. Tiwari, Geophys. Res. Lett. 10, 713-716 (1983).

10. L. W. Alvarez, W. Alvarez, F. Asaro, \& H. V. Michel, Science 208, 1095-1108 (1980). See also L.W. Alvarez, Proc. Natl. Acad. Sci. USA 80, 627-642 (1983).

11. B. Toon et al., in Geol. Soc. Am. Spec. Pap. 190, editors L. T. Silver and P.H. Schultz (Boulder, Colorado, 1982).

12. P. Vail et al., in Seismic Stratigraphy -- applications to hydrocarbon exploration, edited by Charles E. Payton, Memoir 26 of the American Association of Petroleum Geologists (Tulsa, 1977), p. 49-97. See also P. Vail et al., Oceanus 22, 71 (1979).

13. P. Vail, private communication.

14. R. A. Kerr, Science 209, 483-486 (1980).

15. M. Davis, P. Hut, R.A. Muller, Nature 308, 715-717 (1984).

16. D. P. Whitmire and A. Jackson, Nature 308, 713-715 (1984).

17. W. Alvarez and R. A. Muller, Nature 308, 718-720 (1984).

18. A review of the data and refererences to the original sources is found in M. B. Cita, "Messinian Event: A Stratigraphic Case Study" in IUGS Special Publication No 14, edited by E. Seibold and J. D. Meulenkamp (1981?). 